\title{
Emerged optimal distribution of computational workload in the evolution of an undulatory animat
}

\author{
Ben Jones*, Yaochu Jin ${ }^{\$}$, Bernhard Sendhoff ${ }^{\$}$, Xin Yao* \\ *School of Computer Science, The University of Birmingham, UK and ${ }^{\$}$ the Honda Research \\ Institute Europe GmbH, DE
}

\begin{abstract}
The coupling between an agent's body and its nervous system ensures that optimal behaviour generation can be undertaken in a specific niche. Depending on this coupling, nervous system or body plan architecture can partake in more or less of the behaviour. We will refer to this as the automatic distribution of computational workload. It is further automatic since the coupling is evolved and not pre-specified. In order to investigate this further, we attempt to identify how, in models of undulatory fish, the coupling between body plan morphology and nervous system architecture should emerge in several constrained experimental setups. It is found that neural circuitry emerges minimalistically in all cases and that when certain body segmentation features are not coevolved, the agents exhibit higher levels of neural activity. On account of this, it is suggested that an unconstrained body plan morphology permits greater flexibility in the agent's ability to generate behaviour, whilst, if the body plan is constrained, flexibility is reduced with the result that the nervous system has to compensate.
\end{abstract}

\section{Introduction}

In biological organisation, organismic components ('cells', 'tissues', 'organs', 'systems', etc.) are coupled; they are part of a holistic system. A morphological change in one component, either via evolution or during development, will often impact on the organisation of another component. Consequently, major evolutionary transitions have resulted in drastic shifts in body shape and form, underlying component architecture and morphology. This is fundamental since it presents biological organisation as being richly dynamic, survival as being dependent on the interoperability that exists between all morphological aspects (as opposed to between only a few of them); the behaviour that results from this is crucial for the animal's survival. Thus, we must recognise that animal behaviour is ultimately shaped by the coupling existing between its nervous system and body plan morphology. Moreover, the body can actually be thought of as an 'interface' residing between the nervous system and the environment, since in an 'exterior-sense' it is only the body that is directly presented to the environment.

The interacting body then requires an efficient nervous system for control, so that basically, the body can do what it is 'designed' to do. To be efficient, its architecture needs to be coupled to the body in a way that allows for optimal behaviour. Given environmental pressure, the body might evolve in order that it can fit better; and when this happens, the nervous system will commensurately be adapted in order that it can maintain optimal coupling. This means that the coupling itself becomes driven by the 
environment; moreover specifically, it becomes driven by a need to optimise the 'balance' of computational workload existing between the coupled components.

This paper tests the hypothesis that artificial fish-like agents for which certain aspects of the body plan morphology are coevolved, end up, after an evolutionary process, having more minimalistic neural circuitry, and with neural dynamics that engage less in the generation of the behavioural process. Thus we can refer to this as a kind of 'distribution of computational process or workload' hence the title of this paper. Related to this concept is the idea of morphological computation [14,3]. This refers to how in a given agent (natural or artificial), computational process can be offloaded to the passive dynamics of the body plan morphology. Evidence for this is inherent in biology: from architecturally specific central pattern generating circuits [12] through to the fact that in fish locomotion, the size and shape of the fish body can all have an impact on behaviour generation [13,7].

In the creation of a three dimensional fish model, we have been inspired by the pioneering work of Ekeberg $[4,5]$ who modelled undulatory fish such as lamprey, with results comparable to biophysical data, the work of Ijspeert $[8,9]$ who built upon this work by incorporating several artificial evolutionary algorithms into the optimisation of the neural control circuits, and last but not least, the seminal work of Karl Sims [17] who paved a major way in the field of Artificial Life, for the study of embodied cognition.

The work presented in this paper demonstrates a novel framework that can be used to explore the processes by which body and nervous system become coupled during a coevolutionary process. The neural control system in an artificial agent is spatially constrained which puts architecture at the fore of the investigation. Crucially, this means that any behavioural process is largely determined by both the architecture of the nervous system and of the body morphology. Thus the way in which computational workload is distributed will be largely reflected by changes in such architecture. The remainder of this paper is set out as follows. Section 2 introduces a model of an undulatory fish-like agent; this model was used throughout. The experimental setup is outlined in Section 3. Results are presented in Section 4. Discussion and conclusions are then provided in Section 5.

\section{Model}

Geometry A three dimensional wire frame model of a fish has been implemented. The edges of the agent mesh are actually springs obeying Hooke's Laws of spring dynamics. This allows for fluid life-like movements and body contortions. Springs are connected in layers which are then connected to form cuboids. These cuboids, which are representative of body segments, then form the overall agent morphology. See Fig. 1.

Virtual water Given the environment, the animat succumbs to the friction or rather, the drag of the surrounding 'water'. External 'water force' pushes against each face of an animat segment, the magnitude of which depends on the opposing force of the face

pushing back. It is sufficient to use the face's velocity as an approximate measure of 


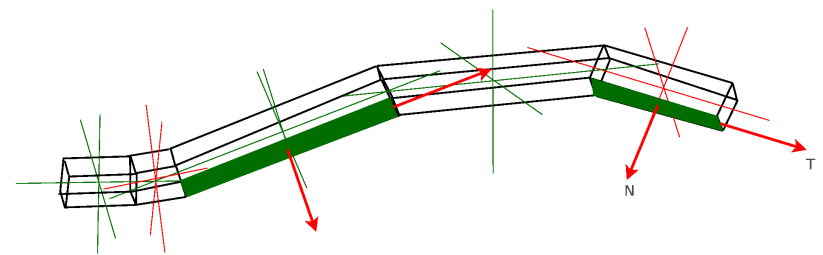

Fig. 1: Agent geometry and highlighted water force model. Each animat segment has a 'compass system' which is used for deriving the directional components of opposing water forces. Two such faces have been filled in with green colour; example vector components used in calculating water force are highlighted with red arrows. ' $\mathrm{T}$ ' represents the tangent component and ' $\mathrm{N}$ ' represents the normal component.

this 'pushing-back' force. The face velocity is taken to be the average over all four constituent point mass velocities (one at each corner of the face). The approach is simple, reliable and efficient in its implementation and has been pursued by others (e.g. [16]).

In terms of the implementation, the face's velocity vector is initially split into two velocity components, the tangent component and the normal component $(t=\hat{\mathbf{t}} \cdot \mathbf{v} ; n=$ $\hat{\mathbf{n}} \cdot \mathbf{v})$. These are represented by ' $\mathrm{T}$ ' and ' $\mathrm{N}$ ' in Fig. 1. The actual water force, w, to be applied to each of the four point masses making up the face is then given as:

$$
\mathbf{w}=\mathbf{f} c d A
$$

where $\mathbf{f}$ is a force vector derived from the normal and tangent velocity components, $c$ is a viscosity coefficient, $d$ is drag, and $A$ is the area of the segment face. The $\gamma$ parameters are set to 1.0 and 0.9 for the normal and tangent components respectively; $c$ and $d$ are further set to 1 .

Neural control system The neural architecture is embedded within the animat wire frame but is intrinsically segmented in the following fashion. Inside of each segment are ten neuron units, four of which are motor neurons and six of which are general interneurons. Spatially, the interneuron positions are randomly distributed and this distribution affects both connection strength and neuron to neuron connectivity. For a given general interneuron, the $(\mathrm{x}, \mathrm{z})$ part of its three dimensional $(\mathrm{x}, \mathrm{y}, \mathrm{z})$ coordinate triplet is initialised in a circular fashion using sine and cosine operations (radii and angle values are uniformally generated). The y-coordinate is then further initialised as a random real value (the animat is initialised in an upright position). These parameters are optimised over a period of simulated evolution.

Connection strengths The weight values are computed from the interneuronal Euclidean distance. The closer two neurons are to each other, the higher the weight value between them, $w_{i j}=\xi / d_{i j}$ where $\xi$ has been empirically set to 20.0 to ensure that a suitable range of weights can be generated.

Connectivity Connectivity also comes about as a function of distance given the sigmoid,

$$
\sigma\left(\lambda, s, d_{i j}\right)=\frac{2}{2+\exp \left((\lambda / s) * d_{i j}\right)}
$$


where $\lambda$ is an evolved parameter, $s$ is a scaling parameter, which is set to the total length of the agent, and $d_{i j}$ is the euclidean distance between neurons $i$ and $j$. A set of four $\lambda$ values is employed each of which caters for one type of connection: $\lambda_{I I}, \lambda_{I E}$, $\lambda_{S E}, \lambda_{A A}$ where I=interneuron, E=effector neuron, $\mathrm{S}=$ sensory neuron; AA indicates connections between interneurons in adjacent segments.

Motor symmetry Body symmetry is also accounted for in the model and is represented by a 'motor symmetry configuration'. Loosely speaking, the motor symmetry configuration determines which motorneurons become driving effectors during movement behaviour (note: motorneurons not emerging as effectors can serve as additional excitatory interneurons, but have no direct influence on movement behaviour). Example motor symmetries are visualised in Fig. 2 a. The motors are actually considered part of the body plan, thus, an emergence in the above property provides a rudimentary means of studying symmetry breaking, a core evolutionary transition of nervous systems (see for example [6]). Note that body symmetry is not investigated in this paper, however, agents will typically emerge to have a bilateral configuration of the type shown in Fig. $2 \mathrm{a}$ (label A). See [10] for an investigation of this.

Computational motor system Each face of a segment has a motor neuron located at its centre. Thus each segment has four motor neurons. In a given segment face, a motor neuron actuates a vertical spring-pair, see Fig. 2b. The amount of force applied to each spring in this pair is proportional to the motor neuron's membrane potential but is normalised to within the range [0,120]; values higher than 120 result in the spring dynamics becoming unstable. Also, a spring is only ever actuated if the motor neuron's membrane potential is between 0 and 1 .

Sensory system The animat has a very rudimentary sensory system consisting of 4 sensory neurons that remain position-fixed at the head of the animat (one at the topmiddle of each segment face). The head segment therefore has 14 neurons rather than the normal 10. Current is injected into all sensors according to the angle of an environmental target object from each.

Computational model The neural system is based on a continuous time recurrent neural network. The membrane potential $u_{j}$ of a neuron is modelled as follows [2]:

$$
\frac{d u_{j}}{d t}=\frac{1}{\tau_{j}}\left(-u_{j}+\sum_{i=1}^{C} w_{j i} a_{i}+I_{j}\right)
$$

where $\tau_{j}$ is a time constant, $w_{j i}$ is a vector of presynaptic connection weights and $I_{j}$ is an external input current. The value $a_{i}$ is a presynaptic neuron's membrane activity computed as $\tanh \left(u_{i}-\beta_{i}\right)$ where $\beta_{j}$ is a bias value. If a neuron is inhibitory then all of its outgoing connection weights are made to be negative.

Evolutionary process An evolutionary algorithm that harnesses both the power of adaptive mutation and local selection is used to evolve all or part of a genotype that consists of neuron positional information, whether or not interneurons are inhibitory, the $4 \lambda$ parameters controlling connectivity levels, motor bias values and motor time constants, interneuron bias values and time constants, the number of body segments, 


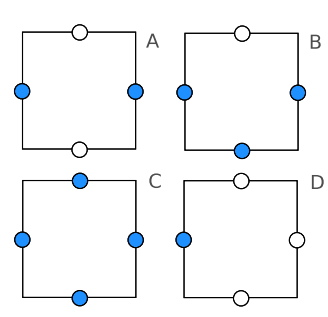

(a)
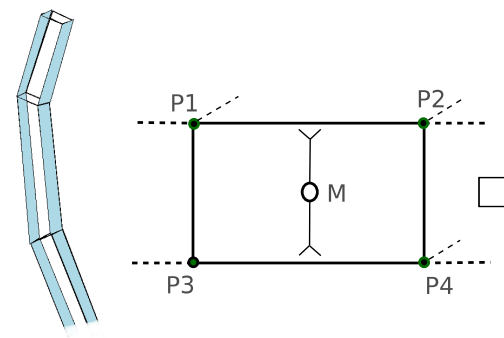

(b)

Fig. 2: (a) Examples of different active motor configurations. Boxes A-D represent example motor symmetries, as viewed from the end of the agent, with filled circles as effectors and unfilled circles as general interneurons. The right hand wire frame diagram exemplifies the 'A' configuration highlighting how both left and right sides become actuated during movement. (b) Motor force system: the effect of a motor $M$ in the centre of one of the segment faces contracting spring $\mathrm{P} 1 \Longleftrightarrow \mathrm{P} 2$ by applying equal but opposite forces $\mathrm{F} 1$ and $\mathrm{F} 2$ to point masses $\mathrm{P} 1$ and $\mathrm{P} 2$ and contracting spring $\mathrm{P} 3 \Longleftrightarrow \mathrm{P} 4$ by applying equal but opposite forces $\mathrm{F} 3$ and $\mathrm{F} 4$ to point masses $\mathrm{P} 3$ and P4.

the lengths of each segment and last but not least, the functional motor symmetry configuration. A local selection process of the type described in [15] is employed, since, (a) preliminary investigation showed it to have far better convergence; (b) within the field of Neuroevolution, a similar strategy has on prior occasion been employed to great success, see for example [18]; (c) the fact that local selection results in gradual phenotypic change is a practical advantage since it allows for evolutionary process to be more easily tracked. Discrete recombination and adaptive mutation which relies on strategy parameters $\tau_{0}=1.0 / \sqrt{2.0 * D}$ and $\tau_{1}=1.0 / \sqrt{2.0 * \sqrt{D}}[1]$ are further applied to the selected chromosome pool with preset probabilities 0.2 and 0.02 respectively.

\section{Experimental setup}

Four sets of 50 differently seeded experiments were conducted; each placed a different level of constraint on the evolutionary process, as described:

1. evo-ALL. The full genotype as described above was evolved.

2. evo-NOBCBL. All parameters except for the number of body segments and the lengths of each segment, were evolved.

3. evo-NOBC. All parameters except for the number of body segments were evolved.

4. evo-NOBL. All parameters except for the lengths of each segment were evolved.

The aim of the first experiment was to essentially discover how 'everything' should become optimally tuned during the evolutionary process. That of the second was to find out how the agent should evolve at the other extreme i.e. when only its nervous system parameters (and body symmetry) are coevolved. The aim of the latter two experiments was to characterise, by counter-example, how changes in segmentation properties (which we may equate to body morphology), affect fitness. In all experiments, 
the agent's task was to swim towards a fixed environmental target placed 20 units away from the head of the agent. Thus fitness was given by the distance of the agent's head from this target as measured at the end of the behavioural sequence; specifically, $20.0-d($ animat, target $)$.

\section{Results}

A visualisation of the behaviour of the best evo-ALL agent is provided in Fig. 3a and plots of fitness are given in Fig. 3b.

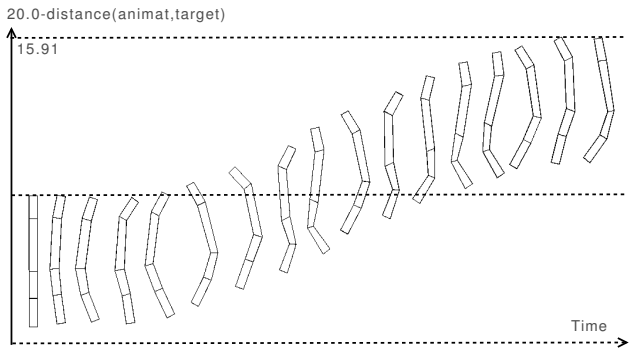

(a)

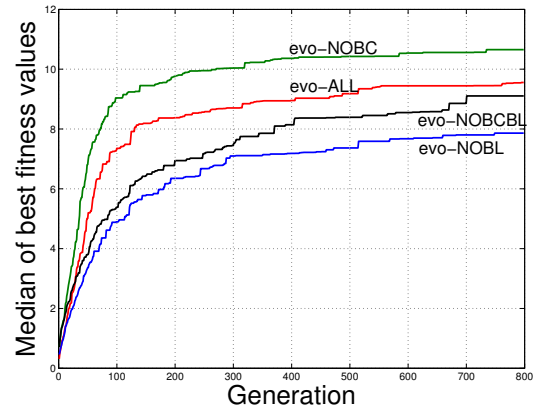

(b)

Fig. 3: (a) Animat behavioural sequences for the best animat from evo-ALL showing undulatory type locomotion in which a wave of propulsion passes down the length of the agent's body. Snapshots taken every 25 behavioural iterations. (b) Medians over best individuals for each of the 4 sets of experiments (median readings are taken to prevent outlier artifacts).

Effect on neural architecture In Fig. 4, the neural architectures for the best individuals to have emerged are visualised. There are several observations to be made. Firstly, there are no connections between the sub-network architectures (one sub-network per body segment). This indicates a preference for fully decentralised, computationally independent sub-networks. Moreover, when connections are artificially added to such agents, fitness decreases in proportion to the number added (results not shown). Secondly, in all cases, few connections were established from the sensory neurons. In instances where connections did emerge, it seems doubtful that the sensory neurons were actually employed, since a simple test in which the target object was removed from the environment subsequent to the evolutionary process found that agents could still successfully locomote. Thus, most agents (if not all) evolved without a true sensory system. Thirdly, the neural architectures typically emerged such that 'functional neurons' - those that became connected within the individual neural circuits - arranged themselves around a 'central axis'. This tendency is highlighted for the four architectures presented in Fig. 4. We can also observe differences in wire length, see Figs. 4e and 4f, with evo-ALL agents evolving to have shorter connections than agents from the other simulations. It would seem that generally, connectivity has a tendency to emerge minimally in all cases and more so in evo-ALL. 
Effect on neural dynamics Statistics of oscillation count (average number of times neuron activation moves from a negative to a positive state, and vice-versa) and motor contraction count ('motor activity', number of motor (spring) compressions), derived from the behaviours of all agents, are plotted in Figs. 5a and 5b. With regards to Fig. $5 \mathrm{a}$, we can observe that neural activity is statistically greater in simulations evo-NOBL and evo-NOBCBL than in evo-NOBC and evo-ALL. Also, with regards to Figs. 5c through 5f, we can observe that actually, neural activity is minimal in all types of agent, given the limited number of neural circuits exhibiting at least some neural activity, but less prominently so in the evo-NOBL and evo-NOBCBL agents. This signifies that for agents in which the lengths of the segments are not evolved, movement kinematics become constrained to the extent that neural activity has to compensate.

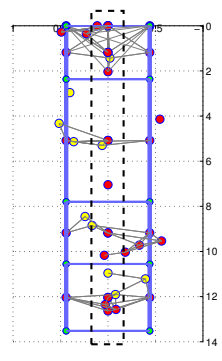

(a) evo-ALL

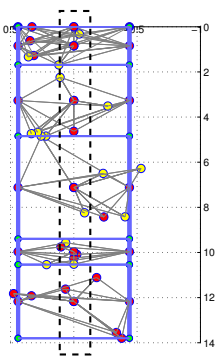

(b) evo-NOBC

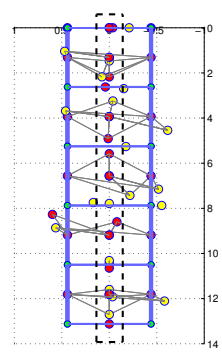

(c) evo-NOBL

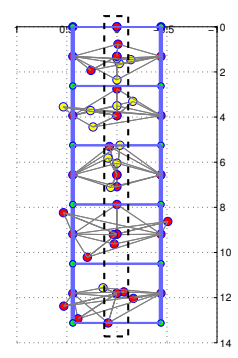

(d) evo-NOBCBL

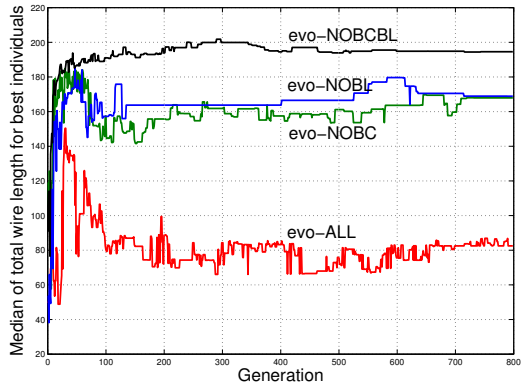

(e) Comparison during evolution.

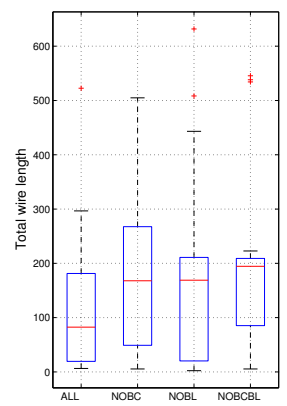

(f) Comparison after evolution.

Fig. 4: (a-d) Best of the best neural architectures. In all architectures, there was a general tendency for neurons to arrange themselves (via evolution of positional information) around a central axis of the agent. These arrangements are highlighted with dashed rectangles. (e and f) wire lengths during and after (boxplot) evolution.

\section{Discussion and conclusions}

The results presented in this paper pave the way in understanding how, for the evolution of a given animal or agent, neural organisation is constrained by the body plan morphology. Successful animal or agent behaviour will only emerge if these two components 
interact appropriately. Evolutionary mechanisms are responsible for tuning this coupling. The results further demonstrate how computational workload can be distributed between body plan morphology and the nervous system.

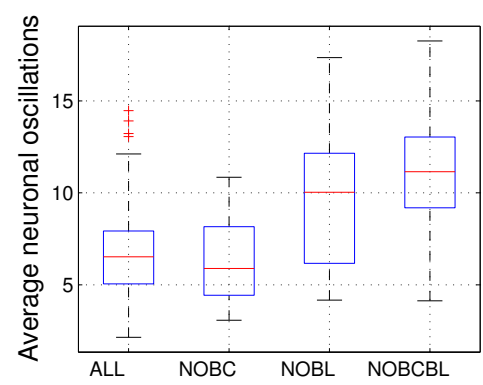

(a)

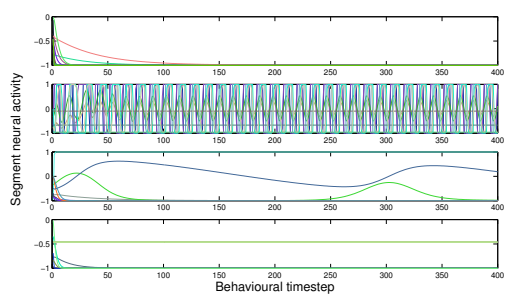

(c) evo-ALL

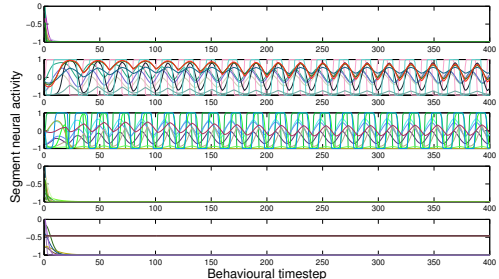

(e) evo-NOBC

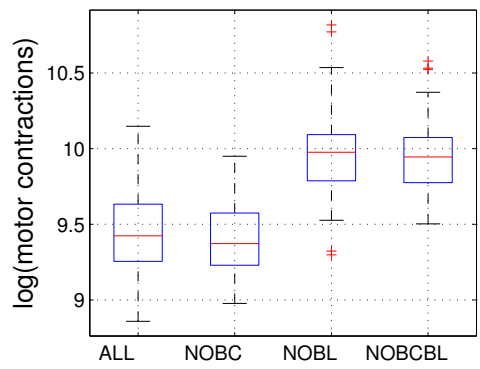

(b)

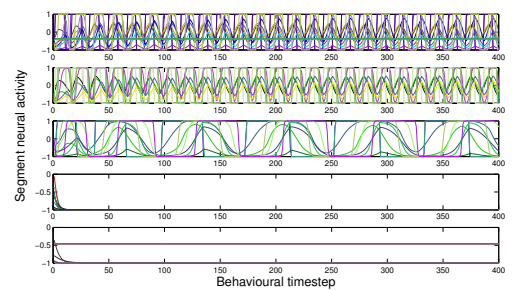

(d) evo-NOBCBL

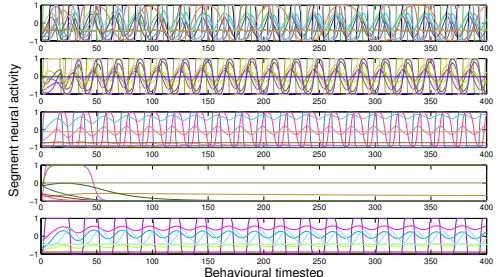

(f) evo-NOBL

Fig. 5: Box plots at the end of evolution for (a) neural and (b) motor activity; (c-f) Neural activities of the very best individuals from each simulation type. Each horizontal bar represents the neural activity in one body segment (the fittest evo-ALL agent emerged to have only 4 body segments).

Let us make light of this. The level of evolutionary pressure exerted on a given component changes throughout the evolutionary process due to the complexity of the fitness landscape; furthermore, the couplings between different components are non-linear and so also succumb to complex selection pressures. Before any evolutionary process has begun (i.e., immediately after random initialisation), all agent types will be subject to equivalent selection forces. Then, as the evolutionary process proceeds forward, the pressures change depending on the pre-existing constraints of the agent. For example, 
in terms of those agents not endowed with segment length coevolution (which were demonstrated to be of significantly worse fitness than all other agent types) we might argue that given minimal flexibility in the evolvability of the body plan morphology, less pressure can and is exerted on this component, however, evolution strives to compensate by commensurately tuning the architecture and computational properties of the nervous system. Thus, we can conjecture that depending on the type of constraint in the body plan morphology - or viewed differently, depending on the level of permitted flexibility - various levels of 'pressure' pre-exist. Based on the constraints set a priori, evolution's search for optimal behaviour is easier or harder from the outset.

The results generally signify three things:

- a tuning of the body plan morphology alone can have a very significant impact on fitness (or, in terms of tuning the number of body segments, a very detrimental impact), and this is before we even consider the computational effort made by the nervous system. In other words, much of the movement control can be offloaded to the dynamics of body morphology; but, in terms of the model, the level of this offloading is potentiated or restricted depending on the constraints that we impose.

- in relation to the above point, when certain features of the nervous system are not coevolved (specifically, the length of each body segment), the neural control system ends up compensating for the lack of flexibility in the body plan. This is demonstrated by higher levels of neural dynamics exhibited in such agents (see Fig. 5). Consequently, such agents can still attain reasonable swimming behaviours.

- minimal neural circuitry was seen to emerge in all agents and in terms of a minimal wire-length, even more so in the evo-ALL agents. This would suggest that when all segmentation characteristics are coevolved, less neural circuitry is required in the generation of correct central pattern generating dynamics.

All three of the above points pertain to the idea that depending on the pre-determined flexibility of the body morphology, when the agent is evolved to reach reasonable levels of behavioural fitness, computational process can be offloaded to different parts of the agent i.e. either to the body plan morphology or to the nervous system (typically in a non-linear combination of both). At the same time, this is demonstrated to have been an automatic process since the way that it should occur is not pre-determined but rather comes about through the evolutionary process.

In conclusion, the integrated coupling existing between body plan morphology and nervous system in an artificial agent lends to a dynamic distribution of computational workload between these two components. Tuning this coupling allows for better distribution; different aspects of the whole agent (nervous system and body plan) are endowed with the capacity to partake in the generation of behaviour. With regard to what is known about such coupling in extant animals, that body symmetry, body shape, and nervous system architecture are all typically 'attuned' to one another (consider for example the Hydra e.g. [11]), it is highly conceivable that such forces and processes are at play in nature.

\section{Acknowledgements}

This work was partially supported by grants from the Honda Research Institute Europe $\mathrm{GmbH}$. 


\section{References}

1. T. Bäck and H.-P. Schwefel. An overview of evolutionary algorithms for parameter optimization. Evolutionary Computation, 1(1):1-23, 1993.

2. J. Blynel and D. Floreano. Levels of dynamics and adaptive behavior in evolutionary neural controllers. In From animals to animats: The seventh international conference on simulation of adaptive behavior, pages 272-281, Cambridge, MA, 2002. MIT Press.

3. J.C. Bongard. Incremental Approaches to the Combined Evolution of a Robot's Body and Brain. PhD thesis, Mathematisch-naturwissenschaftlichen Fakultät der Universität Zürich, Zürich, 2003.

4. Ö. Ekeberg. An integrated neuronal and mechanical model of fish swimming. In Computation in Neurons and Neural Systems, pages 217-222, Washington, DC, 1994. Kluwer.

5. Ö. Ekeberg and S. Grillner. Simulations of neuromuscular control in lamprey swimming. In Philosophical transactions of the Royal Society of London. Series B, Biological sciences, volume 354, pages 895-902. 1999.

6. J.R. Finnerty. The origins of axial patterning in the metazoa: how old is bilateral symmetry? International Journal of Developmental Biology, 47(7-8):523-529, 2003.

7. G.B. Gillis. Undulatory locomotion in elongate aquatic vertebrates: Angulliform swimming since Sir James Gray. American Zoology, 36:656-665, 1996.

8. A. Ijspeert and J. Kodjabachian. Evolution and development of a central pattern generator for the swimming of a lamprey. Artificial Life, 5(3):247-269, 1999.

9. A.J. Ijspeert and M. Arbib. Visual tracking in simulated salamander locomotion. In Sixth International Conference of the Society for Adaptive Behavior, pages 88-97, Cambridge, MA, 2000. MIT Press.

10. B. Jones, Y. Jin, B. Sendhoff, and X. Yao. Evolving functional symmetry in a three dimensional model of an elongated organism. In Artificial Life XI: Proceedings of the Eleventh International Conference on the Simulation and Synthesis of Living Systems, pages 305-312, Cambridge, MA, 2008. MIT Press.

11. O. Koizumi. Developmental neurobiology of hydra, a model animal of cnidarians. Canadian Journal of Zoology, 80(10):1678-1689, 2002.

12. E. Marder and D. Bucher. Central pattern generators and the control of rhythmic movements. Current Biology, 11(23):986-996, 2001.

13. U.K. Müller, J. Smit, E.J. Stamhuis, and J.J. Videler. How the body contributes to the wake in undulatory fish swimming: flow fields of a swimming eel (Anguilla Anguilla). The Journal of Experimental Biology, 204(16):2751-2762, 2001.

14. R. Pfeifer and F. Iida. Morphological computation: Connecting brain, body, and environment. In Biologically Inspired Approaches to Advanced Information Technology, volume 3853, pages 2-3. Springer, Berlin, 2006.

15. J. Sarma and K. De Jong. An analysis of the effects of neighborhood size and shape on local selection algorithms. In Parallel Problem Solving from Nature - PPSN IV, volume 1141, pages 236-244, Berlin, 1996. Springer.

16. M. Sfakiotakis and D.P. Tsakiris. Simuun: A simulation environment for undulatory locomotion. International Journal of Modelling and Simulation, 26(4):350-358, 2006.

17. K. Sims. Evolving virtual creatures. In Proceedings of the 21 st annual conference on Computer graphics and interactive techniques, pages 15-22, New York, NY, 1994. ACM.

18. A. Soltoggio, J.A. Bullinaria, C. Mattiussi, P. Dürr, and D. Floreano. Evolutionary Advantages of Neuromodulated Plasticity in Dynamic, Reward-based Scenario. In Artificial Life XI: Proceedings of the Eleventh International Conference on the Simulation and Synthesis of Living Systems, Cambridge, MA, 2008. MIT Press. 